\title{
NONEQUILIBRIUM RATE THEORY FOR CONDUCTION IN OPEN ION CHANNELS
}

\author{
R. TINDJONG*, I. KAUFMAN, P.V.E. McCLINTOCK \\ Department of Physics \\ Lancaster University, Lancaster, LA1 $4 Y B, U K$ \\ *r.tindjong@lancaster.ac.uk, i.kaufman@lancaster.ac.uk, \\ p.v.e.mcclintock@lancaster.ac.uk \\ D.G. LUCHINSKY \\ Mission Critical Technologies Inc., 2041 Rosecrans \\ Ave. Suite 225 El Segundo, CA 90245, USA \\ Department of Physics \\ Lancaster University, Lancaster, LA1 4YB, UK \\ dmitry.g.luchinsky@nasa.gov \\ R.S. EISENBERG \\ Department of Molecular Biophysics and Physiology, Rush Medical college \\ 1750 West Harrison, Chicago, IL 60612, USA \\ beisenbe@rush.edu \\ Received (received date) \\ Revised (revised date)
}

\begin{abstract}
We present a nonequilibrium reaction rate model of the ionic transition through an open ion channel, taking account of the interaction between an ion at the entrance of the channel and an ion at the binding site in a self-consistent way. The electrostatic potential is calculated by solution of the Poisson equation for a channel modeled as a cylindrical tube. The transition rate, and the binding site occupancy as a function of the left bulk concentration are compared to 1D Brownian dynamics simulations. The analysis is performed for a single binding site of high-affinity, with the exit rate influenced by barrier fluctuations at the channel exit. The results are compared with experimental data for the permeation of the $\mathrm{Na}^{+}$ion through the Gramicidin A channel, with which they are shown to be in good agreement.
\end{abstract}

Keywords: Ion channels, Permeation, Nonequilibrium rate, Stochastic dynamics, Fluctuating barrier

\section{Introduction}

Ion channels are pathways down the centres of proteins embedded into the membranes of biological cells $[1,2]$, facilitating the diffusion of ions across the membranes. They are complicated devices [3], built of thousands of atoms, flexible, and filled with ions and water dipoles that coordinate their movements to allow ions to pass 
one by one across the membrane. To support life, channels have to be precise often selecting between similar ions with an accuracy of 1:1000 [4], yet able to deliver millions of ions per second, i.e. almost at the rate of free diffusion. In recent years impressive progress has been achieved both in understanding the structure of ion channels [3] and in modeling their properties [5]. Yet the mechanism that enables channels to be highly selective while still passing millions of ions per second remains a tantalising paradox.

Channels displaying counter-intuitive behavior of this kind often have one singlyoccupied selectivity site [6] with a high-affinity for a specific type of ion. The fact that a satisfactory explanation of the paradox has yet to be found, even in the most clearly characterised cases [7] of channel conduction, suggests that some basic physical phenomenon has been overlooked. The difficulties in modeling the system stem from the presence of the long-range Coulomb interaction and regions with low and high-dielectric constants. The stochastic dynamics of ionic motion needs to be solved simultaneously with the Poisson equation for all the charges, which often results in strongly nonequilibrium stochastic dynamics. Therefore, only an approximate semi-analytic treatment of the problem is possible.

The simplifying assumptions that are frequently made to overcome these difficulties are sometimes not entirely self-consistent. First note that, in traditional [7-9] reaction rate theory (RRT), transitions of an ion from state $i$ to state $j$ associated with a potential barrier $E_{j i}$ are assumed to proceed at thermal equilibrium with constant rate $k_{j i}$

$$
k_{j i}=\kappa_{0} \exp \left(-\frac{E_{j i}}{k_{B} T}\right)
$$

equal to the product of the frequency factor $\kappa_{0}$ and the Arrhenius factor $\exp \left(-\frac{E_{j i}}{k_{B} T}\right)$, where $k_{B}$ is the Boltzman constant and $T$ is the temperature. At the same time to account for the Born self-energy it is often postulated [10] that an ion can only enter an empty channel: the effect of inter-ion interactions on the escape probabilities is ignored. Secondly, we note that the description of the current of ions through an open ion channel is often described by equilibrium PoissonNernst-Planck (PNP) theory, which also neglects the ion-ion interaction. And the discrepancy between the PNP results and the results of Brownian dynamics (BD) simulations has been the subject of vigorous discussion [11,12]. Yet to explain large ion fluxes through the channels with single high-affinity binding sites, the assumption of multiple-occupancy of the channel is sometimes made [13], ignoring the Born dielectric self-energy altogether. Although the assumptions mentioned may be valid in specific limits of physiological parameters, and helpful in explaining some of the observed experimental data, they are not mutually self-consistent. Being just postulated, rather than related to the channel parameters, they may even be misleading where a different range of physiological parameters is to be considered.

In standard reaction rate theory, ion channels are modeled as a sequence of energy barriers over which the ion must jump. The physical basis of the standard 
three-well model of an ion channel is based on Eyring's transition state theory. The binding sites model postulates that ions pass through channels by binding with one or more particular sites in the channel pore [10]. Läuger went a step further than the Eyring analysis by allowing for the phenomenon of saturation to be observed in his rate theory analysis of the transport [14]. In the binding site model, the net unidirectional flux can be calculated, taking into account the probability that the binding site well is occupied. An ion can enter the well only if it is not already occupied by another ion, and an ion can only leave a well if it is there already, i.e. if the well is occupied. Knowing the probability for the occupied and unoccupied wells is therefore enough to characterize the current.

In this paper we introduce a modified RRT that takes into account the dependence of the escape probabilities on the ion-ion interaction. The theory also illuminates the relation between the PNP and BD results. The electrostatic potential is calculated using the solution of the Poisson equation for a channel modeled as a cylindrical tube. The transition rate and the binding site occupancy as a function of the left bulk concentration are compared to $1 \mathrm{D}$ BD simulations, taking into account particle injection. The analysis is performed for a single binding site of high-affinity, with the exit rate governed by barrier fluctuations. We will compare the model results with experimental measurements of the permeation of $\mathrm{Na}^{+}$ions through the Gramicidin A channel, with which we will show that they are in good agreement.

\section{Rate Model description}

We consider an archetypal model $[1,10]$ of an ion channel with a single high-affinity binding site, as shown in Fig. 1. It is considered as a $30 \AA$ long cylinder of radius $2 \AA$ with a negatively-charged ring at its center. Assuming cylindrical symmetry of the channel, the potential energies for an $\mathrm{Na}^{+}$ion moving along the channel axis for the two state configurations are calculated by solution of Poisson's equation. The transition of an ion e.g. from the left to the right can be described within this model via the following steps (cf. [10]). To be able to enter the channel, an ion must arrive at the channel mouth. It does so with an arrival rate $j_{a r}^{l}$ that is a function of the bulk concentration on the left $C_{l}$, and can be expressed as the average number of arrivals per unit time.

The probability of the ion being in the potential well must be connected to the probability of gaining entrance to the channel when it is empty. This is found by connecting the ion's arrival at the channel mouth to the probability of penetrating the empty channel. An estimate of the arrival time at the channel mouth, assuming charge neutrality and no applied field, can be obtained by considering pure diffusion of ions through an imaginary hemisphere at the mouth of the channel of radius equal to that of the channel. This problem is thus reduced to the determination of the ionic flux through a hemispherical sink at the channel entrance where all ions arriving at the boundary immediately vanish such that the ion concentration there goes to 

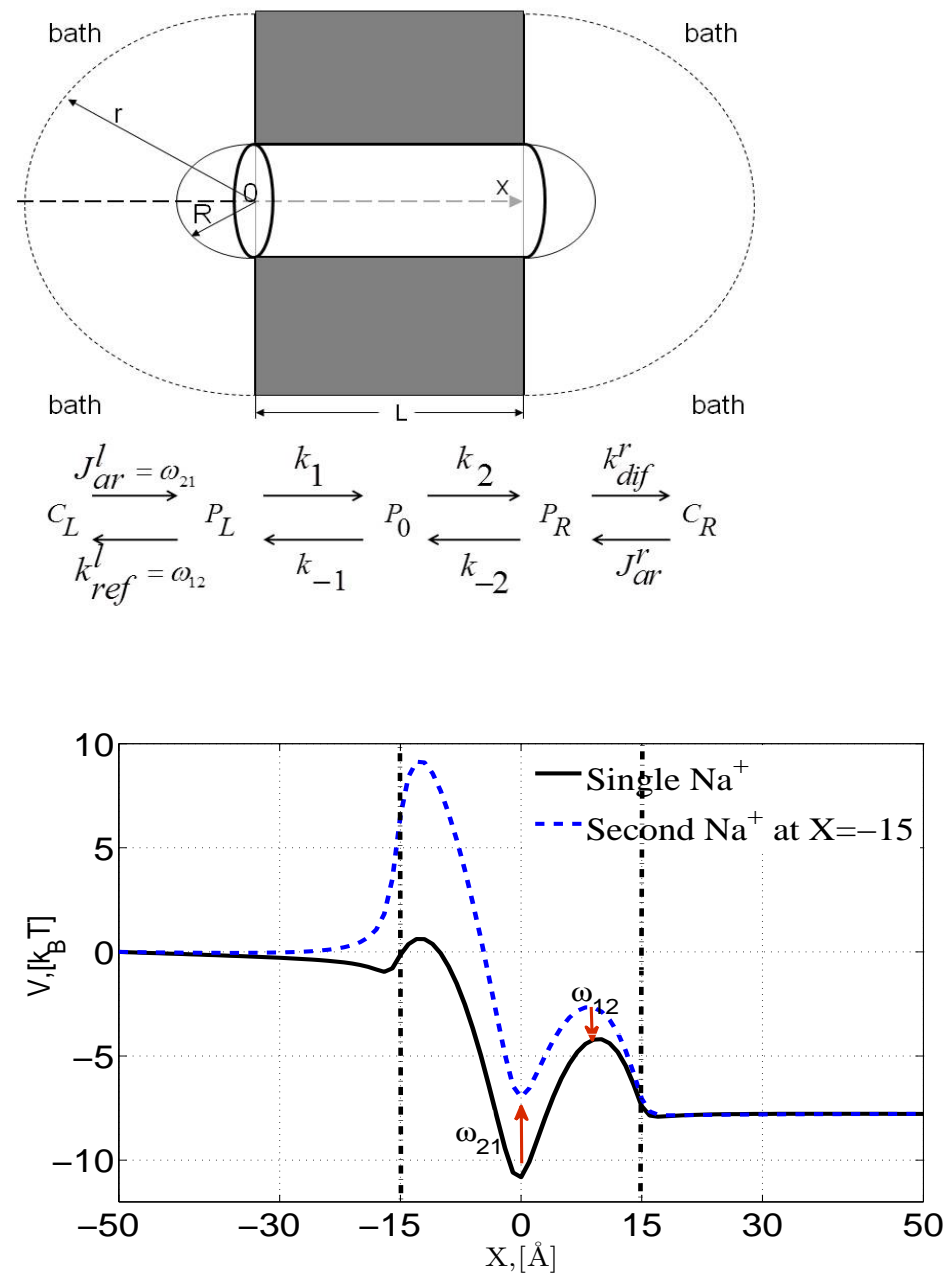

Fig. 1. Top panel: Geometry of the model system under consideration. It is comprised of a cylindrical channel with hemispheres at its left- and right-hand mouths. There is a fixed ring of negative charged (not shown) at its middle. This was the geometry used to solve Poisson's equation. Bottom: Potential energy along the channel axis, for $200 \mathrm{mV}$ applied voltage. The full black line is the potential of a single $\mathrm{Na}^{+}$ion moving along the channel axis. This corresponds to State- 1 . The dashed line is the potential acting on $\mathrm{Na}^{+}$ion moving along the channel axis when there is one $\mathrm{Na}^{+}$ion at the mouth of the channel, corresponding to State-2. The rate of jumping from state-1 to state- 2 is $\omega_{21}=J_{a r}^{l}$, and the rate of jumping from state- 2 to state- 1 is $\omega_{12}=k_{d i f}^{r}$

zero. A first approximation of the rate of arrival at the channel mouth is given by the Smoluchowski rate $1 / \tau_{\text {arrival }}=2 \pi C D R$, where $C$ is the bulk concentration far away from the channel mouth, $D$ is the ion diffusion coefficient and $R$ is the radius 
of the channel.

The bulk concentration is introduced into the model using the injection rate. The fluctuations in the potential energy barrier are the result of ion-ion interactions. We are specifically interested in mechanisms of strong modulation of the potential energy barrier that do not require conformational changes. The rate of barrier modulation is directly related to the rate of ion arrival at the channel mouth. From the left mouth the ion can either diffuse away at diffusion rate $k_{d i f}^{l}$, or bind to the binding site with the rate $k_{1}$. Finally, the ion at the binding site can either return to the left compartment with the rate $k_{-1}$, or exit to the right compartment with the rate $k_{2}$. Similarly, the ion transition from the right to the left is characterized by the set of rates $\left\{j_{a r}^{r}, k_{d i f}^{r}, k_{2}, k_{-2}, k_{-1}\right\}$ and concentration $C_{r}$. The formulation is completed by providing a model for the transition rates and writing a set of kinetic equations, which can readily be solved under conditions of steady current. Therefore, the kinetic equations for the current are given by:

$$
\begin{aligned}
\frac{d P_{l}}{d t} & =J_{a r}^{l}-k_{r e f}^{l} P_{l}-k_{1} P_{l}\left(1-P_{0}\right)+k_{-1} P_{0}, \\
\frac{d P_{0}}{d t} & =k_{1} P_{l}\left(1-P_{0}\right)-\left(k_{-1}+k_{2}\right) P_{0}+k_{-2} P_{r}\left(1-P_{0}\right), \\
\frac{d P_{r}}{d t} & =J_{a r}^{r}-k_{d i f}^{r} P_{r}+k_{2} P_{0}-k_{-2} P_{r}\left(1-P_{0}\right),
\end{aligned}
$$

where $J_{a r}^{l, r}$ are the constant arrival rates at which ions are injected respectively into the left and right volumes located at the mouths of the channel. $P_{l, r}$ are respectively the occupation probabilities of these volumes. $P_{0}$ is the probability of occupation of the charged binding site in the middle of the channel. $k_{r e f}^{l}$ and $k_{d i f}^{r}$ are respectively the diffusive reflection rates on the left and right of the channel. From Eqs. (2-4), the currents to the right and to the left of the channel are given respectively by $J=k_{1} P_{l}\left(1-P_{0}\right)-k_{-1} P_{0}$ and $J=k_{2} P_{0}-k_{-2} P_{r}\left(1-P_{0}\right)$. We are interested in the steady-state current.

The ion injection rate on the left is given by: $J_{a r}^{l}=2 \pi R C_{l} D$, where $C_{l}$ is the concentration in the left bulk. The diffusive and reflection rates at the left and right mouths, $k_{d i f}^{r}$ and $k_{r e f}^{l}$, are calculated from the stationary solution when the channel is closed $(J=0)$. Therefore, the occupation probability of the left hemisphere at the channel mouth is given by $P_{l}=2 \pi R^{3} C_{l} / 3$; corresponding to the number of ions in the left hemisphere. Similar expressions can be written for the ion injection rate and occupation probability of the right mouth, leading to $k_{r e f}^{l}=k_{d i f}^{r}=3 D / R^{2} . P_{0}$ is calculated by combining elements of the steady-state equations. The corresponding equation for $P_{0}$ is quadratic. We are interested in the case of small $k_{2}$ parameters or, in other words in the case when $J \ll J_{a r}^{l}$. The current can then be calculated from:

$$
J=\frac{k_{1}\left(1-P_{0}\right) J_{a r}^{l}-k_{r e f}^{l} k_{-1} P_{0}}{k_{r e f}^{l}+k_{1}\left(1-P_{0}\right)} .
$$

Considering the case of a unidirectional current, the concentration on the right- 
R. Tindjong et al.

hand side of the channel is set to $C_{r}=0$. Since there is no backflow from the right bulk to the left bulk, we set $k_{-2}=0$. The occupation probability at the channel binding site is given by:

$$
P_{0}=\frac{k_{1} J_{a r}^{l}}{k_{1} J_{a r}^{l}+\left(k_{2}+k_{-1}\right) k_{r e f}^{l}} .
$$

The corresponding current is therefore reduced to $J=k_{2} P_{0}$. For a permeating ion of charge $z e$, the current is given by $I=z e J$. The occupation probabilities of the left and right mouth of the channel are given respectively by $P_{l}=\left(J_{a r}^{l}-J\right) / k_{r e f}^{l}$ and $P_{r}=J / k_{d i f}^{r}$. Next, we calculate the rate $k_{2}$ of escape from the single binding site within the framework of fluctuating barrier theory.

\subsection{The ion-ion interaction}

The Poisson Nernst Planck (PNP) approach $[15,16]$ has shed light on how an ion permeates the channel. Nevertheless, being an equilibrium approach, it ignores the ion-ion interaction. To take this into account we consider the changes in potential profile for the ion at the binding site induced by the presence of a second ion at the channel mouth. In this approximation the ion-ion interaction enters the dynamics of the ion inside the channel in the form of a Markovian dichotomous noise. The proposed reaction rate model introduces the rate $k_{2}$ calculated for the case when the barrier fluctuates between two states due to ion-ion interactions. Once the first ion is trapped at the binding site, conduction may occur when a second positive ion arrives at the channel mouth and the system switches to State-2. The latter corresponds to the reduced barrier needing to be overcome by the trapped ion in order to exit to the right bath.

Thus, as the second ion arrives at the channel mouth, the first ion initially at the binding site will have a higher chance of crossing the small barrier and exiting to the right-hand side of the channel, and a much smaller chance of diffusing back to the left bath. Hence the net transport to the right is caused directly by the random (fluctuational) arrival of ions at the left mouth of the channel. To a good approximation, no backflow of ions need be considered and we therefore calculate the unidirectional current.

To describe the two-state potential model, we consider the equation of a pulsating ratchet

$$
m \gamma \frac{d x}{d t}=-\frac{d V(x, \zeta(t))}{d x}+\sqrt{2 m \gamma k_{B} T} \xi(t) .
$$

We thus consider the overdamped unidirectional motion of a $\mathrm{Na}^{+}$ion of mass $m$, with a friction coefficient $\gamma$ which is related to its diffusion coefficient through the fluctuation dissipation relation. The thermal energy of the particle is $k_{B} T$. The potential $V(x, \zeta(t))$ in this approximation is obtained by solving Poisson's equation. It takes account of the following three contributions: (i) the potential of the Coulomb interaction with ions in the bulk; (ii) the electrostatic potential induced by interaction with the protein; (iii) the potential of the Coulomb interaction with 
the wall charge at the selectivity site. This mixed (hybrid) process is governed by $\zeta(t)$; a dichotomous, stationary, Markovian noise and $\xi(t)$; a normalized white noise. For each state of the noise $\zeta(t)$ there corresponds a potential $V_{i}$. The system can be described by the probability distribution $P_{i}(x, t)$, which is the joint probability density for the position $x$ of the particle and the state of the potential $i$. The corresponding Chapman-Kolmogorov equation for this distribution is:

$$
\frac{\partial P_{i}(x, t)}{\partial t}=\frac{\partial}{\partial x}\left(\left[\frac{1}{m \gamma} \frac{d V_{i}}{d x}+\frac{k_{B} T}{m \gamma} \frac{\partial}{\partial x}\right] P_{i}(x, t)\right)+\sum_{j}\left(\omega_{i j} P_{j}(x, t)-\omega_{j i} P_{i}(x, t)\right)
$$

where $\omega_{i j}$ are the transition rates between states $j$ and $i$, given respectively by $\omega_{21}=J_{a r}^{l}$ and $\omega_{12}=k_{r e f}^{l}$. For the two states 1 and 2, Eq. (8) is reduced to:

$$
\frac{\partial}{\partial t}\left(\begin{array}{l}
P_{1}(x, t) \\
P_{2}(x, t)
\end{array}\right)=\left(\begin{array}{cc}
\widehat{L}_{1}-\omega_{21} & \omega_{12} \\
\omega_{21} & \widehat{L}_{2}-\omega_{12}
\end{array}\right)\left(\begin{array}{l}
P_{1}(x, t) \\
P_{2}(x, t)
\end{array}\right)
$$

where the operators $\widehat{L}_{i}$ are given by $\widehat{L}_{i}=\frac{1}{m \gamma} \partial_{x} \frac{d V_{i}}{d x}+\frac{k_{B} T}{m \gamma} \partial_{x}^{2}$. The particle starts at the bottom of the potential (corresponding to the channel binding site $x=x_{0}$ ), with probability $P_{i}(x, 0)=\delta\left(x-x_{0}\right)$. Reflecting boundary conditions are chosen at the bottom of the potential $\left(x=x_{0}\right)$ and absorbing boundary conditions at its top $\left(x=x_{1}\right): \frac{\partial P_{i}}{\partial x}\left(x=x_{0}, t\right)=0 ; P_{i}\left(x=x_{1}, t\right)=0$. Following the standard method described in Gardiner [17] and [18,19], the system of first order ordinary differential equations giving the mean first passage time (MFPT) can be written:

$$
\left\{\begin{array}{l}
\frac{d T_{1}}{d x}=S_{1} \\
\frac{k_{B} T}{m \gamma} \frac{d S_{1}}{d x}=\frac{1}{m \gamma} \frac{d V_{1}}{d x} S_{1}+\omega_{21}\left(T_{1}-T_{2}\right)-1 \\
\frac{d T_{2}}{d x}=S_{2} \\
\frac{k_{B} T}{m \gamma} \frac{d S_{2}}{d x}=\frac{1}{m \gamma} \frac{d V_{2}}{d x} S_{2}+\omega_{12}\left(T_{2}-T_{1}\right)-1
\end{array}\right.
$$

with the boundary conditions $\frac{d T_{i}}{d x}\left(x=x_{0}, t\right)=0 ; T_{i}\left(x=x_{1}, t\right)=0$.

\subsection{Solution of the boundary value problem}

The system Eq. (10) obtained from the fluctuating barrier model is solved numerically as a boundary value problem. The advantages of this method are that it does not require linear approximation of the potential energy and it can easily be extended to a larger number of states; specifically, one needs five states for two types of ions at each side of the channel. Each of the states corresponds to a different potential energy conformation. It is an approach that was not discussed earlier, and one that is easily accessible to a broad audience of potential users.

The MFPT for a particle that starts from the bottom at $x=x_{0}$ is $T=$ $\sum_{i=1}^{2} p^{(i)} T_{i}\left(x=x_{0}\right)$ [20], where $p^{(i)}$ is the occupation probability of the state $(i)$, such that $\sum_{i=1}^{2} p^{(i)}=1$. The rate of escape from the channel binding site to the right bath is given by $k_{2}=1 / T$. 

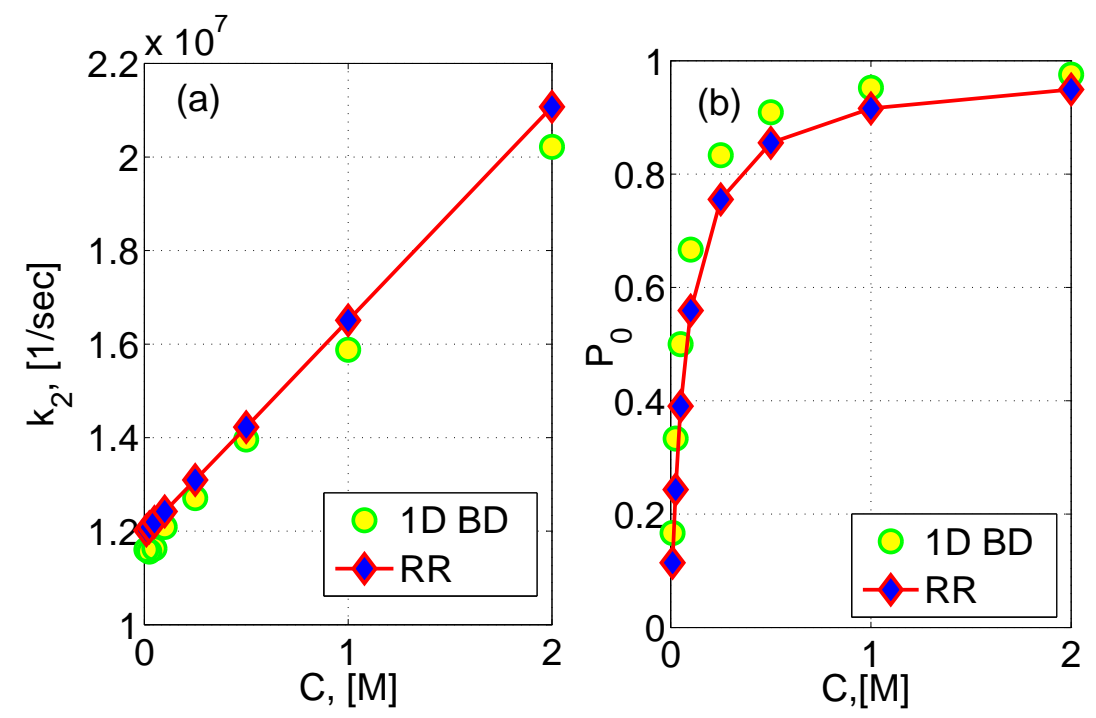

Fig. 2. (a) Escape rate as a function of bulk concentration. The circles are obtained using a onedimensional Brownian dynamics (1D BD) simulation for an $\mathrm{Na}^{+}$inside the channel with particle injection at the channel's left mouth. The lozenges are calculated fluctuating barrier theory. (b) Channel binding site occupancy as a function of bulk concentration. The lozenges are calculated using the modified reaction rate (RR) theory and the circles are obtained from a $1 \mathrm{D}$ BD simulation with ion injection at the channel's left mouth.

\section{Current concentration characteristics}

We have calculated the modified rate $k_{2}$ as a function of the left bulk concentration for comparison with the rate obtained from direct BD simulations, taking into account particle injection at the mouth of the channel with the same Smoluchowski rate. As shown in Fig. 2(a), the two rates follow a similar linear dependence as a function of the concentration and are in reasonable agreement (cf. the Kramers rate over a non-fluctuating energy barrier which is independent of the concentration). The channel occupancy is also calculated and compared to that obtained from BD simulations: see Fig. 2(b). We compare the results with Andersen's experimental data [21] for the current of $\mathrm{Na}^{+}$ions in a gA channel in Fig. 3; we used the rates $k_{1}=$ $3.3 \times 10^{10} \mathrm{~s}^{-1}, k_{-1}=1.2 \times 10^{7} \mathrm{~s}^{-1}$ and a diffusion coefficient of $D=1.17 \times 10^{-9} \mathrm{~m}^{2} \mathrm{~s}^{-} 1$ for the $\mathrm{Na}^{+}$ion.

\section{Summary and perspectives}

We have introduced a non-equilibrium rate model of the ionic transition through an open ion channel within a self-consistent framework. The method takes explicit account of the interaction between an ion at the left mouth and an ion at the channel 


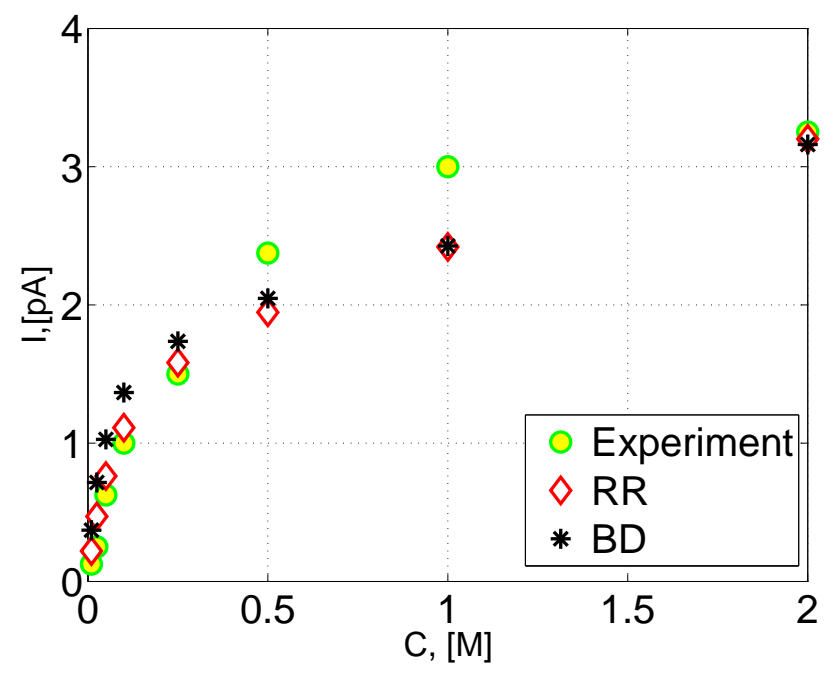

Fig. 3. Current as a function of concentration for an $\mathrm{Na}^{+}$ion in the $\mathrm{gA}$ channel. The circles are experimental data from Andersen [21]. The lozenges represent the results of calculations based on the modified rate model. The stars are obtained from a $1 \mathrm{D}$ BD simulation for an $\mathrm{Na}^{+}$inside the channel with particle injection at the channel left mouth.

binding site. These ion-ion interactions result in fluctuations of the potential energy barrier of an ion at the binding site calculated from the channel structure by solving Poisson's equation. We calculated the transition rate and binding site occupancy of an $\mathrm{Na}^{+}$ion as a function of the left bulk concentrations, and compared the result with 1D BD simulations using ions injection at the channel mouth. The model was applied to experimental permeation rate data for $\mathrm{Na}^{+}$ions in the Gramicidin A channel, yielding satisfactory agreement. Note that, in the case of Gramicidin, the ion-ion interaction is not particularly important, so that equilibrium PNP theory may be sufficient. We expect, however, that the effect will be of crucial importance in channels that display both high conductivity and high selectivity, e.g. the $\mathrm{K}^{+}$ and $\mathrm{Ca}^{2+}$ channels. These will be analyzed in our future work. Note also that the boundary value technique used for solution of the MFPT system of equations can readily be extended to encompass a larger number of states. This feature will be needed e.g. for the case when there are ions at both channel mouths, which five states will be required.

\section{Acknowledgements}

The research was supported by the EPSRC, UK. 


\section{References}

[1] B. Hille, Ionic Channel Of Excitable Membranes (Sinauer Associates, Sunderland, MA, 2001), 3rd edition.

[2] R. S. Eisenbeg, "Ions in fluctuating channels: transistors alive", Fluct. and Noise Lett., in this issue.

[3] J. D. Stockand and M. S. Shapiro, Ion Channels: Methods and Protocols (Humana Press, Totowa, N.J., 2006).

[4] D. A. Doyle, J. M. Cabral, R. A. Pfuetzner, A. Kuo, J. M. Gulbis, S. L. Cohen, B. T. Chait and R. MacKinnon, "The structure of the potassium channel: Molecular basis of $\mathrm{k}^{+}$conduction and selectivity", Science 280 (1998) 69-77.

[5] S.-H. Chung, V. Krishnamurthy and O. S. Andersen, Biological Membrane Ion Channels Dynamics, Structure, and Applications (Springer, 2007).

[6] P. Hess and R. W. Tsien, "Mechanism of ion permeation through calcium channels", Nature 309 (1984) 453-456, 10.1038/309453a0.

[7] E. W. McCleskey, "Calcium channel permeation: A field in flux", J. Gen. Physiol. 113 (1999) 765-772.

[8] P. C. Jordan, "Ion permeation and chemical kinetics", J. Gen. Physiol. 114 (1999) 601-604.

[9] O. S. Andersen, "Perspectives on ion permeation", J. Gen. Physiol. 113 (1999) $763-$ 764 .

[10] D. J. Aidley and P. R. Stanfield, Ion channels: Molecules in action (Cambridge University press, Cambridge New York Melbourne, 1996).

[11] G. Moy, B. Corry, S. Kuyucak and S.-H. Chung, "Tests of continuum theories as models of ion channels. I. Poisson-Boltzmann theory versus Brownian dynamics", Biophys. J. 78 (2000) 2349-2363.

[12] B. Corry, S. Kuyucak and S.-H. Chung, "Tests of continuum theories as models of ion channels. II. Poisson-Nernst-Planck theory versus Brownian dynamics", Biophys. J. 78 (2000) 2364-2381.

[13] C. M. Armstrong and J. Neytonc, "Ion permeation through calcium channels", Annals of the New York Academy of Sciences 635 (1991) 18-25.

[14] P. Läuger, "Ion transport through pores: A rate-theory analysis", Biochimica et Biophysica Acta (BBA) - Biomembranes 311 (1973) 423-441.

[15] M. G. Kurnikova, R. D. Coalson, P. Graf and A. Nitzan, "A lattice relaxation algorithm for 3d poisson-nernst-planck theory with application to ion transport through the gramicidin a channel", Biophysical Journal 76 (1999) A211-A211, part 2.

[16] B. Corry, S. Kuyucak and S.-H. Chung, "Tests of Poisson-Nernst-Planck theory in ion channels", J. Gen. Physiol 114 (1999) 597-599.

[17] C. Gardiner, Handbook of Stochastic Methods, for Physics, Chemistry and the Natural Sciences Second Edition. (Springer, Berlin Heidelberg New York Barcelona Hong Kong London Milan Paris Tokyo, 1983).

[18] M. Bier and R. D. Astumian, "Matching a diffusive and a kinetic approach for escape over a fluctuating barrier", Phys. Rev. Lett. 71 (1993) 1649-1652.

[19] C. R. Doering and J. C. Gadoua, "Resonant activation over a fluctuating barrier", Phys. Rev. Lett. 69 (1992) 2318-2321.

[20] J. H. Li, D. Y. Xing, J. M. Dong and B. Hu, "Resonant activations for a fouctuating barrier system driven by dichotomous noise and gaussian white noise.", PRE 60 (1999) 1324-1328.

[21] O. S. Andersen, R. E. Koeppe and B. Roux "Gramicidin channel", IEEE Trans. Nanosci. 4 (2005) 10-20. 